\title{
Introduction to the Second Special Issue
}

The call for papers for the second special issue on mobilizing the mental health strategy for Canada, Changing Directions, Changing Lives (the Strategy), was met with an overwhelming response. This suggests a growing mental health research enterprise in Canada that has the potential to assist in mobilizing the Strategy.

At the same time, the mental health research community in Canada is mourning the loss of two of its outstanding members. Dr. Paula Goering and Dr. Elliot Goldner were both exceptional researchers in their own right, and provided unparalleled mentorship to new generations of mental health researchers who continue to "carry the torch." In this second special issue, we present a tribute to Dr. Goering, written by one of her many mentees, Dr. Elizabeth Lin, and a tribute to Dr. Goldner, former Chair of the MHCC Scientific Advisory Committee, by MHCC President and CEO, Louise Bradley.

With so many thought-provoking articles, we are publishing the second special issue in two parts. This first part of the second issue includes articles that address Strategy themes pertaining to leadership, health promotion, diversity, partnerships with service users and their families, as well as collaboration with primary care. Many of the articles focus on the priority area of mental health of children and youth. Now that five years have passed since the Strategy's release, we see an increasing number of articles that focus on the effective implementation of the ideas contained within the Strategy put into practice.

From a leadership perspective, we begin with an article by Bartram and Lurie, who describe the need for public policy leaders to take advantage of a new window of opportunity to close the gap in public mental health spending as a proportion of overall health costs between Canada (7\%) and other countries (9\%) and relative to the global mental health disease burden, estimated at $13 \%$ globally. The authors describe policy options for spending the five billion dollars in new federal funding available over the next 10 years, as the latest twist in "the long and winding road" to close mental health funding gaps in Canada.

Mulvale and colleagues next discuss the MHCC's leadership in hosting a consensus conference in November 2015, as a way to share best evidence and stimulate new thinking about how to address the mental health needs of "emerging adults" (adolescents and young adults). The authors discuss what was heard in follow-up interviews with participants about their experiences at the conference and whether, and if so, how it helped them move evidence from the conference into practice. National level consensus conferences, they suggest, are helpful as a knowledge exchange approach in a decentralized context like Canada, and a means to catalyze action by provincial and territorial governments, health regions, and community organizations. Lessons learned for future consensus conferences are presented.

Three articles speak to the theme of health promotion and the need for community supports. Kerman and colleagues use survey data from 96 housing and 186 community-based mental health service providers in a pan-Canadian research project on housing and supports for people with mental illness, to explore the unmet support needs, barriers, and facilitators to helping this population to become and stay housed, as seen by service providers. Both groups highlight the need for more safe, affordable housing with supports, as well 
as the development of service partnerships to reduce the burden and barriers faced by direct-service providers in addressing the unmet support needs of people with mental illness to help them stay stably housed. Pakzad and colleagues share the results of the Moncton site of the At Home Chez Soi project, which implemented a Housing First intervention using a multi disciplinary team. The study found no difference in the use of emergency and ambulatory care between the intervention group and the control group, but a lower rate of psychiatric hospitalizations at 24 months. Kanagaratnam and colleagues re-examine current approaches to mental well-being and illness in the immigrant and refugee population arriving from war torn countries and identify challenges in contemporary clinical practice among mental health practitioners in comparison to emerging literature in this field. In doing so, the article seeks to bridge the gap between existing knowledge and current practice, and provides recommendations for mental health practitioners who work with this population.

Three articles highlighted the importance of youth engagement at various levels, for various purposes: design of resources, implementation, and improving service journeys. Huggett and colleagues shine a spotlight on youth engagement in a system-level partnership initiative to develop resources that are relevant to youth's own needs and preferences. In this case the partnership led to the development of an app called Be Safe that is a resource that helps youth manage mental health crises and identify local services. The example provides lessons learned for other initiatives and points to how successful youth engagement led to the development of a resource that has garnered attention from organizations and communities across Canada. Brownlie and colleagues focus on the importance of youth engagement in the review of youth substance abuse services including services addressing concurrent substance use and mental disorders in Ontario. The authors discuss the important role of youth as collaborators as well as respondents, and the processes used to tailor findings to local contexts, in moving from consultation to implementation of system enhancements recommended in the review. Tilleczek and colleagues explore the conceptual literature on youth journeys in mental health and present examples from Atlantic Canada that illustrate how journey mapping approaches can assist in engaging young people and attend to their journeys of healing and recovery.

Danseco and colleagues further elaborate approaches to support implementation of evidence-informed practices to children and youth through the People Advancing Change through evidence (PACE) program by community agencies in Ontario. This spotlight article provides a concrete example of how to integrate implementation drivers, organizational factors, change management and project management approaches based on the implementation science literature. The authors share project leader perspectives on successes, challenges and lessons learned.

Engaging users as partners and the importance of collaborative approaches across sectors, but in particular between primary care and mental health services were also important themes. In their case study that shines a spotlight on a novel form of wraparound model that features family peer support Olibris and colleagues present the Caroline Families First Program as an exemplar of how several of the Strategy's strategic directions (cross-sectoral collaboration and planning, peer support, person/family directed care) can be realized in practice. Key themes that arose from interviews with a wide range of program participants were the importance of building family capacity, family centredness and engagement, and paid family peer support for families with children with complex mental disorders. The Olibris study also notes the 
importance of co-location of the program within a primary health care centre as facilitator of collaborative mental health care.

Finally Alain Lesage and colleagues call for the development of a pan Canadian strategy for suicide prevention which would feature a population focus, improved access to services including psychotherapy, establishment of targets and improved surveillance and quality control.

Several common themes that arise across all of these articles are the need for multi-sectoral collaboration, community focused services, improved access to housing, supporting transitions for emerging adults from child to adult mental health and substance abuse services, fully engaging people receiving services and their families, and adopting evidence-based approaches to implementation. We hope the articles will challenge your thinking about approaches to mobilizing Canada's mental health strategy. As you read the articles, we encourage you to reflect on how actions underway in your jurisdiction align with the Strategy's goals and recommendations.

We would like to thank John Sylvestre, Jean Hughes, Howard Chodos, Tania Lecomte, and Christopher Canning, for their support and encouragement. Thanks also to our reviewers, Katherine Stewart and the journal's editorial team for their support of this project.

Gillian Mulvale and Steve Lurie 